\title{
INTEGRATION OF GRAPHIC AND SEMANTIC INFORMATION IN GEOGRAPHIC INFORMATION SYSTEMS OF DISTRIBUTION ELECTRIC NETWORKS
}

\author{
Viktor Yarosh ${ }^{1}$, Sergei Oskin ${ }^{2}$, Alexey Efanov ${ }^{1}$, Vitaly Shemyakin ${ }^{1}$ \\ ${ }^{1}$ Stavropol State Agrarian University, Russia; \\ ${ }^{2}$ Kuban State Agrarian University named after I.T. Trubilin, Russia \\ yarviktor@yandex.ru,kgauem@yandex.ru,yefanov@mail.ru, shi_ma@mail.ru
}

\begin{abstract}
One of the important and promising areas for increasing the efficiency of managing the operation of distribution electric networks is the use of geographic information systems (GIS). In geographic information systems, graphical and associated with it attributive (semantic) information is used. Maps, plans, diagrams with distribution electric networks printed on them are used as graphic information in geographic information systems. Databases of electrical equipment are used as semantic information for the description of distribution electric networks. Thanks to the joint processing of graphic and semantic information in geographic information systems, the efficiency of all stages of working with spatial data is increased. Starting from the search, selection and analysis of spatial data to the development of a specific solution for managing the operation of distribution electric networks. The article analyzes the feasibility of using a formal number as a unique identifier. Three approaches to the organization of the joint work of graphic and semantic information in geographic information systems have been considered. Structural schemes, advantages and disadvantages of ways of integrating graphic and semantic information have been given. The features of integration of graphic and semantic information in geographic information systems of distribution electric networks have been also considered. The prospects of using an object-oriented approach to organizing the joint work of graphic and semantic information in geographic information systems have been noted.
\end{abstract}

Keywords: geographic information systems, electric power network, integration, spatial data.

\section{Introduction}

The advantage of GIS is the linking of graphic (spatial) and attributive (semantic) data. The attributive description supplements the spatial description, together with it creates a complete description of GIS models, solves the problems of typing source data, and simplifies the classification and processing processes [1-4]. The classification of attribute information is presented in Table 1.

Table 1

\section{Classification of attribute information}

\begin{tabular}{|l|l|}
\hline Attribute type & \multicolumn{1}{c|}{ Attribute value } \\
\hline Primary & Measured, entered \\
\hline Secondary & Obtained by calculation from the values of other attributes \\
\hline Classical & Alphanumeric \\
\hline Extended & Multimedia \\
\hline Generalized & Performing actions \\
\hline Singular & One-of-a-kind \\
\hline Plural & Has a temporal or statistical characteristic \\
\hline
\end{tabular}

Initially, attributes could be primary (measured, entered) and secondary, calculated, obtained by calculation from the values of other attributes. For example, the active and reactive resistances of power lines are calculated based on the primary attributes of specific electrical resistance and line lengths.

Today, in the era of multimedia, it is quite natural to expand the concept of an object's attributes to other information related to it: bitmap graphics, an object's photo, diagram, a video film, computer animation, sound information. Therefore, we can talk about the classical (alphanumeric) attributes of the object, as well as its extended attributes (multimedia, in particular).

In modern GIS, it is possible to associate some action with an object, for example, launch some program or macro, when an object is activated by pointing to it. It is also possible, when activating an object, for example, an image of a substation on a schematic map of the main network, to switch to another scheme, which is the operational scheme of the substation $[5,6]$. Thus, the operational scheme of the substation can be considered as an attribute of the first object. This makes it necessary to 
introduce the concept of "generalized attributes" and clearly demonstrate the conventionality and relativity of dividing information about an object into attribute and non-attribute in GIS.

Attribute data may have temporal characteristics. The time response characteristic can be reflected in several ways:

- by correlating information with certain points in time;

- by specifying the time period of the existence of objects;

- by specifying the speed of objects.

Depending on the way the time characteristic is reflected, it can be placed in one table or in several attribute tables of a given object for different time steps.

The use of attributes allows the analysis of database objects using standard query forms and various filters, as well as expressions of mathematical logic.

The relationship of coordinate and attributive descriptions can be organized in different ways. For example, W. Weber proposed a specific combination of coordinate and attribute classes to describe cartographic data [7]. To build a general GIS data model, he introduced a four-dimensional object space, where the first two sizes were assigned to $\mathrm{X} / \mathrm{Y}$ data, attributes were located in the third dimension, and the fourth dimension was reserved for temporary data sets. In most modern GIS, the association of graphic and attribute information is defined as:

- unambiguous (one to one) relationship between map objects and entries in the table of object attributes;

- the relationship between the object and the record is maintained using a unique identifier assigned to each object;

- the unique identifier is physically stored in two places: in files containing pairs of $\mathrm{x}, \mathrm{y}$ coordinates, and in the corresponding entry in the object attribute table.

\section{Materials and methods}

All objects and primitives must have their own identifier; with which semantic information to them can be bound. Using an identifier opens up wide possibilities for viewing and analysis. The user can select an object on an electronic map, and the system will determine its identifier, by which it will find all the characteristics related to the object stored in one or more databases. Conversely, by selecting information about objects that satisfy a certain condition in the tables, the user can immediately see the location of these objects on an electronic map.

An identifier is necessary; otherwise the object is not unique and cannot be interpreted as fully individual. Possible identifier options are presented in Table. 2.

A unique name can be used as an identifier, for example, a unique name that is not repeated anywhere else within the study area (or part of it), for which it is necessary to observe the uniqueness of identifiers. In general, using only names as identifiers is not very convenient and reliable. In Russia there are several rivers with the name Bystraya and there are several cities with the name Paris on the globe.

Table 2

Identifiers

\begin{tabular}{|c|l|l|}
\hline Type & \multicolumn{1}{|c|}{ Explanation } & \multicolumn{1}{|c|}{ Notes } \\
\hline Name & $\begin{array}{l}\text { A name that is not repeated within the scope of } \\
\text { the study (or part thereof) }\end{array}$ & $\begin{array}{l}\text { The existence of objects with the } \\
\text { same name. }\end{array}$ \\
\hline Coordinates & $\begin{array}{l}\text { The object has a certain location, position in } \\
\text { space. }\end{array}$ & $\begin{array}{l}\text { An object may change its position } \\
\text { over time. Dependence on the } \\
\text { used coordinate system. }\end{array}$ \\
\hline Number & $\begin{array}{l}\text { A formal number assigned automatically in the } \\
\text { process of entering graphic information or } \\
\text { assigned in the process of setting semantic } \\
\text { information. }\end{array}$ & $\begin{array}{l}\text { The use of the number is most } \\
\text { appropriate as a unique identifier. }\end{array}$ \\
\hline
\end{tabular}


An individual object has a certain location, position. This positional information, however, is inconvenient for use as an identifier. An object, both natural (river) and technogenic (transformer) can change its position over time. In addition, positional information differs in accuracy (instrument and measurement method), and it also differs due to the use of that other coordinate system.

The most appropriate is to use a number as a unique identifier. For example, the object number on some list or cadaster. Most GISs use a formal number as a unique identifier, assigned by the program automatically in the process of entering graphic information, or can be assigned in the process of setting semantic information.

Usually, semantic information is organized in the form of tables that operate with a special, very complex set of programs called a database management system (DBMS). In the simplest case, each map object in the image of the electronic map is assigned a row in the table, where the semantic information is recorded.

A table containing attributes of objects is called an attribute table. Each spatial object corresponds to a row in the table; to each feature, a column in the table. Each cell of the table reflects the value of a particular attribute for a particular object.

\section{Results and discussion}

There are three approaches (models) to the organization of joint work of graphic and semantic (attributive) information (principles of GIS interaction with the database). These approaches (Table 3) are associated with the development of DBMS capabilities [8-10].

Models for organizing joint work of graphic and semantic information

Table 3

\begin{tabular}{|c|l|}
\hline Type & \multicolumn{1}{c|}{ Explanation } \\
\hline $\begin{array}{c}\text { Georelational (hybrid } \\
\text { or mixed) }\end{array}$ & $\begin{array}{l}\text { Separate storage of graphic and semantic information. Graphic } \\
\text { information is stored in proprietary files or GIS file systems, semantic in } \\
\text { a database. Between them, a connection is established through the } \\
\text { identifier of the object. }\end{array}$ \\
\hline Integrated & $\begin{array}{l}\text { It provides for the use of DBMS tools for storing both graphic and } \\
\text { attribute information. }\end{array}$ \\
\hline Object-oriented & $\begin{array}{l}\text { Not points, lines, polygons and records are stored in relational tables, but } \\
\text { objects and methods of interaction between objects. }\end{array}$ \\
\hline
\end{tabular}

The first model, the most famous and widespread today, is georelational, sometimes also called hybrid or mixed. This model uses separate storage of graphic and semantic databases (GDB and SDB). Graphic information, and in some systems also topological, is stored in its own files or GIS file systems. Semantic information is stored in a database (usually relational). Between them, connections are simply established and maintained through the object identifier, Figure 1.

Semantic information is organized into tables that are managed using a relational DBMS. This DBMS can be either integrated into GIS software as its functional subsystem, or it can be external in relation to GIS. Sometimes, both approaches are implemented simultaneously. There is a simple builtin subsystem, and it is possible to use external DBMSs for storing semantic information.

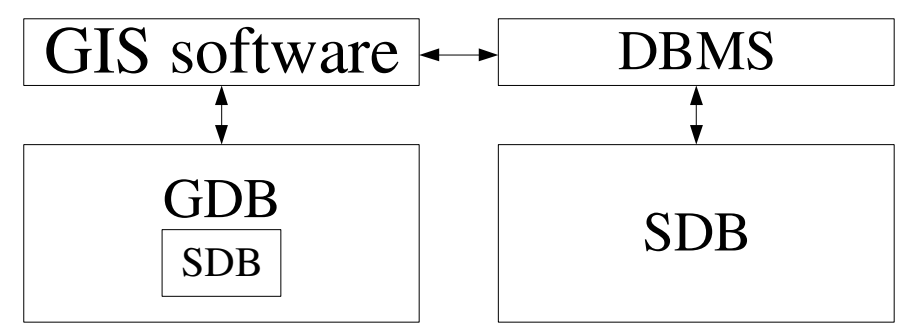

Fig. 1. Georelational model

This approach is based on the fact that it was difficult to achieve simultaneous optimization of storage of both graphic and semantic data. In addition, in many GIS applications, there is a tendency to 
complete GIS to large databases existing under DBMS management, adding spatial components and appropriate means of working with it.

The second model is an integrated one. It provides for the use of relational DBMS tools for storing both graphical and attribute components. In this case, the GIS acts as a kind of add-on over the DBMS (Figure 2). That allows to search and analyze simultaneously on both characteristics. For processing both spatial and semantic data, a single access method is used, which is the SQL query language.

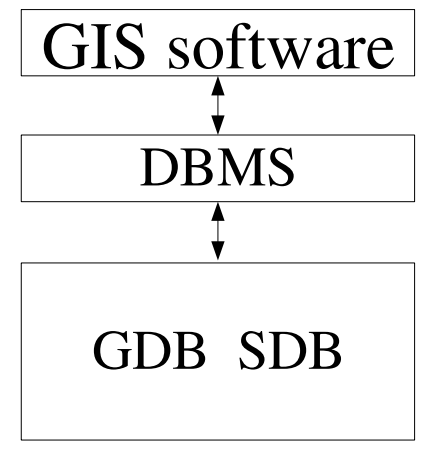

Fig. 2. Integrated model

This approach has several advantages, especially for large information repositories operating in an active multi-user mode, when ensuring data integrity becomes a significant problem. However, modern traditional relational DBMSs were not very suitable for working with spatial objects of a general form (other than point objects). The transition from GDB file storage to DBMS is a completely natural way of developing GIS. The leadership here undoubtedly belongs to the spatial cartridge of Oracle. There are other storages built on the basis of various DBMSs and implementing various data storage models.

The third model is object-oriented. It has many attractive sides in terms of the possibility of describing very complex data structures in it, the relationships between objects and the ability to solve numerous modeling problems.

Object orientation is not always understood here in the sense that GIS is written with objectoriented tools, but in the fact that the same mechanisms should be provided to the user of the system for organizing data. Object-oriented data models allow to simultaneously simulate the state and "behavior" of objects in GIS. The structure of an object-oriented GIS model is shown in Figure 3.



Fig. 3. Object-oriented model

When using object-oriented GIS data models, it becomes easier to use, the software will work with concepts that are natural to a user, such as a support, switch, power line, etc., and not with system-oriented concepts such as points, lines, polygons. The true potential of object technology lies in the fulfillment of the task of modeling and sequential analysis, which is simpler, more consistent with the way people normally think. This model is less common due to the difficulty of organizing the entire system of relationships between objects.

The attributes for the objects of electric distribution networks can be the source data for calculating the distribution network mode 


$$
D_{R C P}=\left[P a s, T I_{G}, Y, S_{\text {Load }}\right],
$$

where Pas - passport data of power lines and transformers needed to calculate the equivalent circuit parameters;

$T I_{G}-$ network topology information;

$Y$ - vector of adjustable parameters;

$S_{\text {Load }}$ - node load vector.

Obtaining topological information about the graph $G$ object $j$ of the electrical network connection diagram from the image of the GIS object $j$ can be written in the following form:

$$
V_{o b j G I S} \rightarrow T I_{o b G} \text {. }
$$

For the entire network (2), it can be written:

$$
V_{G I S} \rightarrow T I_{G},
$$

where $V_{G I S}-$ image of an electric network in a GIS.

The equation (2) for the entire electrical network can be written as:

$$
\left[P a s, V_{G I S}, Y, S_{\text {Load }}\right] \rightarrow\left[P a s, T I_{G}, Y, S_{\text {Load }}\right] \text {. }
$$

These equations (3) or (4) in particular describe the integration of GIS and routine calculation programs (RCP) of distribution networks.

The solution to the problem of integrating GIS with RCP is possible in two ways:

1. By the use of information exchange between GIS database and RCP database of electrical networks;

2. By embedding the design modules of the RCP of electrical networks in GIS software.

Also, as an attribute, the results of the calculation of the modes of electric networks and the errors of the calculation results from inaccurate data input can be saved [11].

\section{Conclusions}

1. Modern GIS should support an integrated model for organizing the collaboration of graphic and semantic information.

2. Particularly promising is the use of an object-oriented model in a GIS that uses not points, lines, polygons and records in relational tables, but objects and methods of interaction between objects.

3. The equations of integration of GIS and RCP of distribution networks are given.

\section{References}

[1] Yarosh V. A., Efanov A.V., Yastrebov S.S., Privalov E.E. Presentation of spatial data of electric networks in geo-information systems. Engineering for Rural Development. Latvia University of Life Sciences and Technologies. 2019. Vol. 18. pp. 1661-1666.

[2] Harder C. Enterprise GIS for energy companies. Published by Environment System Research Institute, Inc. ESRI Press. 1999. 110 p.

[3] Meehan B. Empowering Electric and Gas Utilities with GIS. Published by Environment System Research Institute, Inc. ESRI Press. 2007. 280 p.

[4] GIS Best Practices. GIS for Renewable Energy. January 2010. ESRI Press. 46 p.

[5] Efanov A.V., Oskin S.V., Khorolsky V.Ya, Yarosh V.A., Shemyakin V.N. Geo-information system in the intellectual network management structure. Research Journal of Pharmaceutical, Biological and Chemical Sciences. 2018. Vol. 9. No. 6. pp. 1582-1587.

[6] Wong P. C. et al., "A Novel Visualization Technique for Electric Power Grid Analytics," in IEEE Transactions on Visualization and Computer Graphics, vol. 15, no. 3, pp. 410-423, May-June 2009.

[7] Weber W. Drei Typen geographischer Datenstructuren $\square$ Gemeinsamkeiten. Unterschiede und eine mögliche Synthese // Nachr. Karten- und Vermessungsw. 1978. Bd. 1. N 75. S. 133-157. 
[8] Rezaee N, Nayeripour M, Roosta A, Nikna T. Role of GIS in distribution power systems. World Academy of Science, Engineering and Technology, vol. 36 (2009) pp. 361-365.

[9] Parikh P. A. Nielsen T. D. "Transforming traditional geographic information system to support smart distribution systems," 2009 IEEE/PES Power Systems Conference and Exposition, Seattle, WA, 2009, pp. 1-4.

[10]Datta A. Mohanty P., "Enterprise GIS and Smart Electric Grid for India's power sector," 2013 IEEE PES Innovative Smart Grid Technologies Conference (ISGT), Washington, DC, 2013, pp. 1-7.

[11] Efanov A.V., Yastrebov S.S., Atanov I.V., Sharipov I.K. Analysis of the error in calculating the mode in the problem of eliminating accidents in distribution electric networks // Research Journal of Pharmaceutical, Biological and Chemical Sciences. 2018. Vol. 9. No. 4. pp. 727-733. 